\title{
Vitamin A metabolism in chick liver: some properties of the cytosolic lipid-protein aggregate
}

\author{
BY D.SKLAN AND ORNA HALEVY \\ Faculty of Agriculture, Hebrew University, Rehovot 76-100, Israel
}

(Received 20 September 1983 - Accepted 4 January 1984)

\begin{abstract}
1. Incubation of hepatic microsomes with retinol resulted in formation of retinyl esters and glucuronides. The presence of the cytosolic lipid-protein aggregate (LPA) in the system in addition induced release of holoretinol-binding protein from the microsomes. The extent of these reactions was influenced by the addition of coenzyme A and ATP, or uridine diphosphate glucuronic acid.

2. Incubation of hepatic microsomes containing labelled retinyl esters with the LPA resulted in the appearance of the labelled retinyl esters in the LPA.

3. Small amounts of retinoic acid were formed on incubation of retinol with microsomes (approximately $1 \%$ of added retinol); this was found to be associated with a protein of approximately 14500 molecular weight, and less than $10 \%$ was associated with the LPA. This is in contrast to retinol, which was found to be almost completely associated with the LPA.

4. The cytosolic LPA was associated both with carotene-cleavage activity and alcohol dehydrogenase $\left(\mathrm{NAD}(\mathrm{P})^{+}\right)(E C 1.1 .1 .71)$ activity.

5. These findings lend some support to the concept of a specific role for hepatic LPA.
\end{abstract}

A high molecular weight (MW) lipid-protein aggregate (LPA) containing retinyl esters and retinol has been described in the cytosol of tissues of several animals (Heller, 1979; Sklan et al. 1982; Sklan \& Donoghue, 1982a, b). This LPA comprised about $50 \%$ lipids and had a hydrated density in the range 1.052-1.13 and co-eluted with zinc- and coppercontaining proteins (Chen \& Heller, 1979; Sklan et al. 1982; Sklan \& Donoghue, 1982a,b). The latter appear to form part of an enzyme or enzymes with acyl glyceride and retinolpalmitate palmitohydrolase (EC 3.1.1.21) activities. The intracellular retinol-binding protein (cRBP) was associated with the LPA and could be dissociated from it on incubation at $37^{\circ}$ or on treatment with detergent (Sklan et al. 1982; Sklan \& Donoghue, $1982 \mathrm{~b}$ ). Furthermore, carotene-cleavage enzymes appeared to be associated with a similar fraction in the intestinal mucosal cytosol (Sklan, 1983). The LPA thus appears to be connected with several functions of hepatic vitamin A metabolism; however, it is by no means clear whether the LPA represents a specific lipoprotein particle or, at the other extreme, a non-specific hydrophobic aggregate. The present study attempted to elucidate further some of the functions of the LPA.

\section{EXPERIMENT AL}

$\beta-\left[15,15^{\prime}-{ }^{3} \mathrm{H}\right]$ carotene, $\left[11,12-{ }^{3} \mathrm{H}\right]$ retinoic acid and $\left[15-{ }^{14} \mathrm{C}\right]$ retinol were gifts from HoffmanLa Roche (Basle). $\beta$-Carotene, retinal, retinol and retinoic acid were obtained from Sigma Chemical Co. Inc. (St Louis, Mo) and were purified by alumina chromatography (Goodman et al. 1967) just before use. Livers were obtained from 2-4-week-old male chicks, homogenized in a high-speed homogenizer and subcellular fractions and cytosol prepared as previously described (Adachi et al. 1982). The LPA was prepared by gel filtration on a Sepharose 4B column $(500 \times 20 \mathrm{~mm})$, eluting with Tris-hydrochloride buffer, $\mathrm{pH} 8 \cdot 0$, in dim light at $4^{\circ}$ (Sklan et al. 1982). Additional gel filtrations with Sepharose 6B $(470 \times 17 \mathrm{~mm})$ or Sephadex G-50 $(500 \times 20 \mathrm{~mm})$ columns were carried out under similar conditions (Sklan \& Donoghue, 1982a). Columns were calibrated with protein standards as previously described (Sklan et al. 1982; Sklan \& Donoghue, 1982a). 
Gel-permeation chromatography was carried out using a TSK $3000(300 \times 7.5 \mathrm{~mm} ; \mathrm{LKB}$, Bromma) column eluting with $0.1 \mathrm{M}$-phosphate buffer, $\mathrm{pH} 6 \cdot 8$, containing $0.1 \mathrm{M}$-sodium chloride; detection was with a variable-wavelength u.v.-visible detector (Jasco Corp., Tokyo). The column was calibrated with blue dextran, apoferritin, phosphorylase (EC 2.4.1.1), albumin, ovalbumin, myoglobin, ribonuclease (EC 3.1.4.22) and bovine pancreatic trypsin inhibitor. Reverse-phase chromatography was carried out with a Partisil ODS 2 column ( $250 \times 4.1 \mathrm{~mm}$; HPLC Technology, Macclesfield), eluting with $8 \%$ aqueous methanol, and straight-phase chromatography with a Partisil ODS 10 column (HPLC Technology) eluting with $3 \%$ dioxane in hexane. Ultracentrifugation was performed with a Beckman L3-50 centrifuge with a Ti 50 rotor, and fluorescence was determined with a Perkin Elmer 203.

Carotene-cleavage activity was determined essentially as described by Goodman $e t$ al. (1967) and Fidge et al. (1969). Alcohol dehydrogenase (EC 1.1.1.1) activity was determined by following the change in concentration of NADH as described by Vallee \& Hoch (1955). Alcohol dehydrogenase $\left(\mathrm{NAD}(\mathrm{P})^{+}\right)(E C 1.1 .1 .71)$ activity was determined by extracting the reaction products and subjecting the extract to straight-phase highperformance liquid chromatography (HPLC) for quantitative determination of retinol and retinal.

Esterification of retinol, retinyl glucuronide production and holo-retinol-binding protein (holo-RBP) release from microsomes were determined by incubation of microsomes resuspended in $0.1 \mathrm{M}$-phosphate buffer, $\mathrm{pH} 7.5$, in the presence of various additives. The incubation was terminated by exhaustive extraction and retinol and retinyl esters quantified by reverse-phase HPLC. An additional portion of the reaction mixture was incubated for a further 10 min with 100 units $\beta$-glucuronidase $(E C 3.2 .1 .31)$ before extraction and HPLC. The efficiency of release of retinol by this procedure was over $90 \%$. Glucuronides were calculated by determining the difference in the retinol concentration between portions of the reaction mixture before and after glucuronidase incubation (Sklan, 1983). A third portion of the reaction mixture was recentrifuged at $105000 \mathrm{~g}$ for $60 \mathrm{~min}$ and RBP determined in the supernatant by TSK 3000 gel permeation (additional details of RBP identification are given in the results section). RBP was determined in the microsomes after extensive homogenization and incubation with retinol prior to gel permeation chromatography. In some experiments, microsomes were incubated with ${ }^{14} \mathrm{C}$-labelled retinol for 30 min before addition of LPA. In experiments to determine transfer of retinyl esters to the LPA, incubation was terminated by two-layer sucrose density centrifugation, the density of the top layer was 1.07 and that of the bottom layer 1.16; the top layer contained the LPA.

A liver cytosol fraction rich in CRBP was prepared by incubating liver cytosol with $\left[{ }^{14} \mathrm{C}\right] \mathrm{retinol}$; the $\mathrm{pH}$ was then reduced to $5 \cdot 0$. Following filtration of the cytosol, gel filtration on a G-75 column at $\mathrm{pH} 8.0$ was carried out and the labelled fraction eluting at $14000-15000$ daltons was collected (Ong et al. 1978).

RESULTS

LPA was prepared from liver cytosol by gel filtration on a Sepharose 6B column as previously described (Sklan et al. 1982). This fraction on Sepharose 4B chromatography had an elution volume very close to that of blue dextran, and had a hydrated density in the range 1.063-1.21.

Microsomes prepared from liver by differential centrifugation were incubated in the presence of retinol added in ethanol solution. Under these conditions esterification of retinol and production of glucuronides was observed (Table 1). Both these processes could be 
Table 1. Changes $(\Delta)$ in concentrations $(\mu \mathrm{g})$ of retinyl esters $(R E)$, retinyl glucuronides and retinol-binding protein $(R B P)$-retinol on incubation of hepatic microsomes with different additives

(Results are means and standard deviations of six determinations, and are expressed as the increase in concentration of the respective fraction compared with incubation of hepatic microsomes with buffer only. All additives contained $5 \mu \mathrm{g}$ retinol equivalents, and incubation was for $1 \mathrm{~h}$ at $37^{\circ}$. CoA and uridine diphosphate glucuronic acid (UDPGA) were added to give $2 \mathrm{~mm}$ final concentration, and ATP to $10 \mathrm{~mm}$ final concentration. Quantification of retinyl esters was by reverse-phase high-performance liquid chromatography (HPLC), retinyl glucuronides by HPLC before and following glucuronidase (EC 3.2.1.31) incubation, and RBP-retinol following $105000 \mathrm{~g}$ recentrifugation by gel permeation HPLC. Total RBP in the system (microsomes + supernatant fraction) was $8.6 \mu \mathrm{g}$.)

\begin{tabular}{|c|c|c|c|c|c|c|}
\hline \multirow[b]{2}{*}{ Additive } & \multicolumn{2}{|c|}{$\Delta \mathrm{RE}$} & \multicolumn{2}{|c|}{$\begin{array}{c}\Delta \text { Retinyl } \\
\text { glucuronides }\end{array}$} & \multicolumn{2}{|c|}{$\begin{array}{c}\Delta \text { Supernatant } \\
\text { RBP-retinol }\end{array}$} \\
\hline & Mean & SD & Mean & SD & Mean & SD \\
\hline Retinol & 0.25 & 0.03 & $0 \cdot 27$ & 0.02 & $0 \cdot 04$ & $0 \cdot 01$ \\
\hline Retinol + CoA - ATP & 0.42 & 0.05 & $0 \cdot 20$ & 0.05 & -0.02 & 0.00 \\
\hline Retinol + UDPGA & 0.08 & 0.01 & 0.82 & 0.09 & -0.01 & 0.00 \\
\hline Retinol + UDPGA + CoA - ATP & $0 \cdot 30$ & 0.07 & 0.63 & 0.07 & 0.05 & 0.01 \\
\hline Retinyl palmitate & $-0 \cdot 02$ & 0.01 & 0.03 & $0 \cdot 00$ & 0.02 & 0.00 \\
\hline LPA & $0 \cdot 16$ & 0.02 & $0 \cdot 24$ & 0.05 & $0 \cdot 46$ & 0.07 \\
\hline $\mathrm{LPA}+\mathrm{CoA}-\mathrm{ATP}$ & 0.39 & 0.04 & 0.21 & 0.04 & $0 \cdot 14$ & 0.02 \\
\hline LPA + UDPGA & -0.02 & 0.00 & $1 \cdot 18$ & $0 \cdot 08$ & 0.06 & 0.02 \\
\hline $\mathrm{LPA}+\mathrm{UDPGA}+\mathrm{CoA}-\mathrm{ATP}$ & $0 \cdot 31$ & 0.06 & 0.72 & 0.05 & 0.04 & 0.02 \\
\hline cRBP & 0.01 & $0 \cdot 01$ & 0.01 & $0 \cdot 00$ & 0.09 & 0.03 \\
\hline LPA lipids & 0.08 & 0.02 & 0.09 & 0.03 & 0.01 & 0.01 \\
\hline
\end{tabular}

LPA, lipid-protein aggregate; cRBP, intracellular RBP.

enhanced by adding either coenzyme $\mathrm{A}(\mathrm{CoA})$ and $\mathrm{ATP}$, or uridine diphosphate glucuronic acid (UDPGA). Addition of both additives together also resulted in enhancement of both esterification and glucuronide production. On incubation of the hepatic microsomes in the presence of LPA, an additional process was observed which involved release of protein-bound retinol to the incubation medium. Further examination of this moiety by gel-permeation chromatography showed that the retinol-protein complex had a MW of approximately 20000 daltons. This material exhibited a fluorescence spectrum with an excitation maximum at approximately $340 \mathrm{~nm}$ and an emission maximum at $460 \mathrm{~nm}$. When the incubation procedure was carried out with labelled retinol, and the retinol-protein complex isolated and incubated with a 500 -fold excess of cold retinol, over $80 \%$ of the label was displaced from the protein complex. These findings together suggest that the retinol was bound to R BP. The rate of appearance of this fraction is shown in Fig. 1. Addition of CoA and ATP, or UDPGA, or both to the microsomes incubated with LPA reduced the amount of RBP released (Table 1). Retinol, retinyl palmitate or the lipids extracted from the LPA were not effective in releasing RBP. A cRBP-rich fraction of liver cytosol did cause some release of RBP, but this was about $20 \%$ of that obtained with a similar amount of retinol in the LPA. The amount of RBP released per hour was approximately $5 \%$ of the total microsomal RBP.

In some experiments microsomes were pre-incubated with retinol in ethanol for $20 \mathrm{~min}$ and LPA was then added and incubation continued. Under these conditions, labelled retinyl esters were observed in the LPA in amounts increasing with time and with the amount of LPA added (Fig. 2).

In addition to the formation of retinyl esters and glucuronides on incubation of 


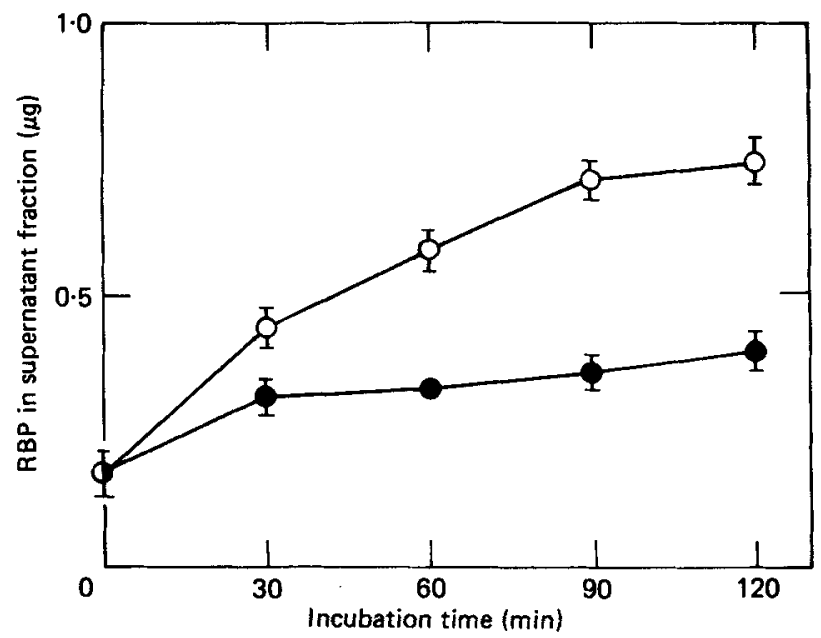

Fig. 1. The change in retinol-binding protein (RBP) concentration in the $105000 \mathrm{~g}$ supernatant fraction on incubation of hepatic microsomes for $1 \mathrm{~h}$ with either $(O)$ lipid-protein aggregate $(5 \mu \mathrm{g}$ retinol equivalent) or (O) with an equal volume of buffer. Points are mean values and standard deviations are represented by vertical bars.

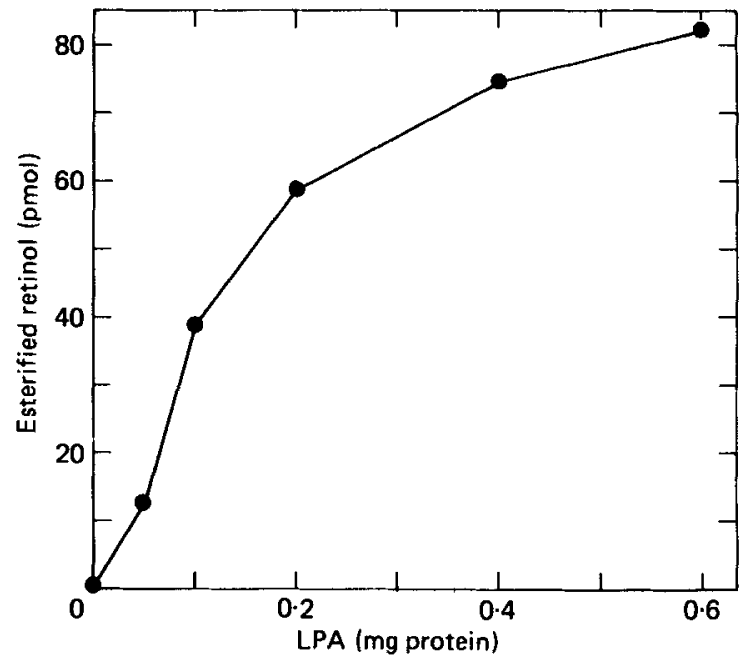

Fig. 2. Appearance of labelled retinyl esters in lipid-protein aggregate (LPA) following addition of different amounts of LPA to microsomes preincubated with ${ }^{14} \mathrm{C}$-labelled retinol. Following incubation the samples were centrifuged at $105000 \mathrm{~g}$ in sucrose layers, LPA was separated and retinyl esters determined following alumina chromatography.

microsomes with retinol in ethanol, small amounts of polar material co-eluting with retinoic acid on reverse-phase HPLC were detected. This comprised some $1 \%$ of added retinol. In order to attempt to determine the transport vehicle for retinoic acid, liver cytosol was incubated with $\left[{ }^{3} \mathrm{H}\right]$ retinoic acid and subjected to gel filtration on a Sepharose $6 \mathrm{~B}$ column. The elution profile is shown in Fig. 3 and is compared with that of $\left[{ }^{14} \mathrm{C}\right]$ retinol treated in the same manner. The major proportion of retinoic acid eluted with an apparent $\mathrm{MW}$ of 14000-15000 daltons, with small amounts associated with the LPA. This is in contrast to retinol which was almost completely observed in the LPA. 


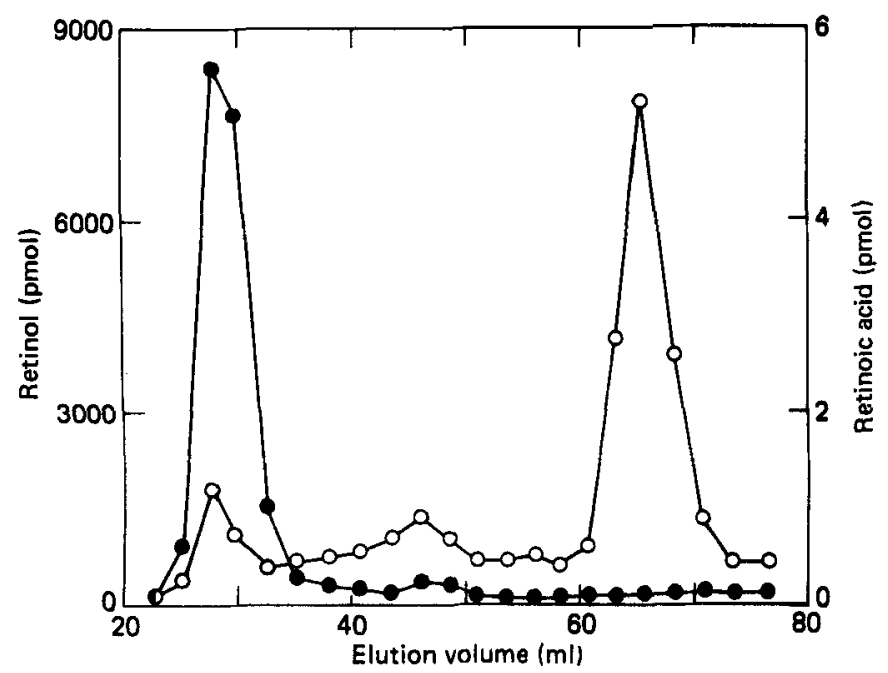

Fig. 3. Sepharose $6 \mathrm{~B}$ elution profile of hepatic cytosol incubated with $\left[{ }^{3} \mathrm{H}\right] \mathrm{retinoic}$ acid $(\mathrm{O})$ and $\left[{ }^{14} \mathrm{C}\right] \mathrm{retinol}(\mathrm{O})$. Cytosol was prepared by $105000 \mathrm{~g}$ centrifugation of a $1: 1 \mathrm{high}$-speed homogenate of chick liver. Cytosol $(0.6 \mathrm{ml})$ was loaded on to the column and fractions of approximately $2.3 \mathrm{ml}$ collected.

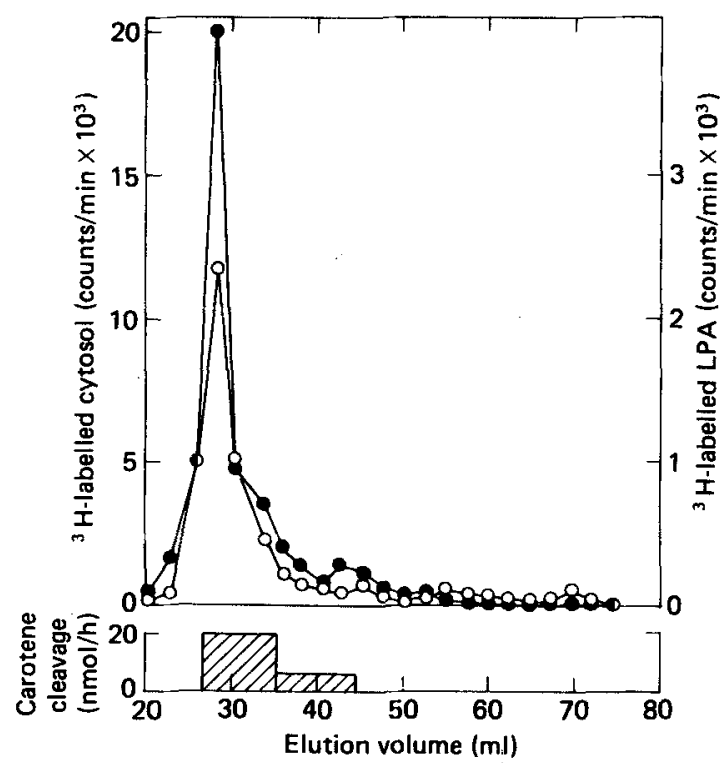

Fig. 4. Sepharose 6B elution profile of hepatic cytosol incubated with $\beta-\left[{ }^{3} \mathrm{H}\right]$ carotene $(O)$ or of lipid-protein aggregate (LPA) (O) isolated from a Sepharose 4B column. (国), Carotene-cleavage activity in the eluent. Cytosol was prepared by $105000 \mathrm{~g}$ centrifugation of a $1: 1$ high-speed homogenate of chick liver. Cytosol $(0.6 \mathrm{ml})$ was loaded on to the column and fractions of approximately $2.3 \mathrm{ml}$ collected.

Liver cytosol was incubated with ${ }^{3} \mathrm{H}$-labelled $\beta$-carotene and subjected to Sepharose $6 \mathrm{~B}$ gel filtration and the label was determined in the eluent (Fig. 4). Virtually all the added label eluted in the LPA. Determination of the carotene-cleavage activity in the eluent fractions revealed that carotene-cleavage activity co-eluted mainly with the LPA (Fig. 4). In addition, liver cytosol incubated with carotene was chromatographed on a Sepharose 4B column and the LPA isolated rechromatographed on the Sepharose 6B column; LPA co-eluted with 
the peak observed by direct chromatography. LPA was ultracentrifuged using the $\left[{ }^{3} \mathrm{H}\right]$ carotene as a marker and $2.4 \%$ of the added label were found in the floating top fraction at a density of 1.063 in comparison with $94.3 \%$ at a density of 1.21 .

Alcohol dehydrogenase activity was observed in the LPA fraction and, on incubation with acetaldehyde and NADH, ethanol was formed at a rate of 12.4 (SD 1.5$) \mu \mathrm{mol} / \mathrm{min}$ per $\mathrm{mg}$ protein. Some reaction was also observed in the absence of exogenous NADH. The reverse reaction was observed in the presence of ethanol and NAD, although at a lower rate $(1 \cdot 2$ (SD 0.3$) \mu \mathrm{mol} / \mathrm{min}$ per mg protein). Incubation of retinal and NADH with LPA resulted in retinol formation $(2.6 \mu \mathrm{mol} / \mathrm{min}$ per $\mathrm{mg}$ protein) and again some reaction was observed in the absence of exogenous $\mathrm{NADH}$. These activities were inhibited by 1,10-phenanthroline ( $2 \mathrm{~mm}, 58 \%$ inhibition) and 5',5-dithiobis-(2-nitroenzoic acid) (4 mM, $81 \%$ inhibition). Conversion of retinol to retinal in the presence of NAD was not detected.

Incubation of the LPA followed by rechromatography on Sepharose 6B showed partial dissociation of the LPA; determination of alcohol dehydrogenase activity in the eluent revealed activity in the LPA and at a MW in the range 70000-90000 daltons (values not shown).

\section{DISCUSSION}

The findings presented in the present study indicate that the LPA is involved in the intracellular transport of both retinol and retinyl esters as well as with carotene transport and cleavage. Incubation of retinol with hepatic microsomes has been previously reported to result in the production of both retinyl esters and retinyl glucuronides (Lippel \& Olson, 1968; Sklan, 1983). The hepatic esterification appears to be via a fatty acyl coenzyme A: retinol acyl transferase which is enhanced in the presence of CoA and ATP (Ross, 1982). The extent to which esterification and glucuronide production proceeded appeared, under the conditions used in the present study, to be interdependant with the amount of product as influenced by the level of cofactors present. An additional process involving the release of protein-bound retinol from the microsomes was observed in the presence of LPA and, to a lesser extent, cRBP. This protein, on the basis of the MW, fluorescence spectrum and specificity of retinol binding appeared to be RBP. In preliminary studies with rat hepatic microsomes, the release of RBP following LPA incubation was established by radioimmunoassay (D. Sklan, W. Blaner, N. Adachi, J. E. Smith and D. S. Goodman, unpublished results). Addition of retinol, retinyl ester or the lipids of the LPA was not effective in releasing the microsomal $\mathrm{RBP}$, and it thus appears that the transfer of retinol to RBP may require specific proteins. Chen et al. (1981) showed that, in vitro, the LPA can transfer retinol to RBP in the presence of albumin. Quantitatively, it appears that the amount of release of holo-RBP from the microsomes is influenced by the extent of esterification and glucuronide production occurring concurrently. It could thus be speculated that the release of holo-R BP from the liver could be influenced by the availability of cofactors for esterification and glucuronide production.

Incubation of the LPA with microsomes containing labelled retinyl esters resulted in the appearance of some of these retinyl esters in the LPA. This indicates that LPA may play a role in retinyl ester transport.

As previously reported (Lippel \& Olson, 1968) some retinoic acid was formed on incubation of liver microsomes with retinol. Transport of retinoic acid in liver cytosol appeared to be predominantly in a protein complex of a MW of 14000-15000 daltons, presumably the intracellular retinoic-acid-binding protein (Ong \& Chytil, 1975). This was in contrast to retinol which was associated almost exclusively with the LPA in cytosol either as the ester or as cRBP. A possible scheme for some of these reactions in hepatic vitamin A metabolism is given in Fig. 5. 


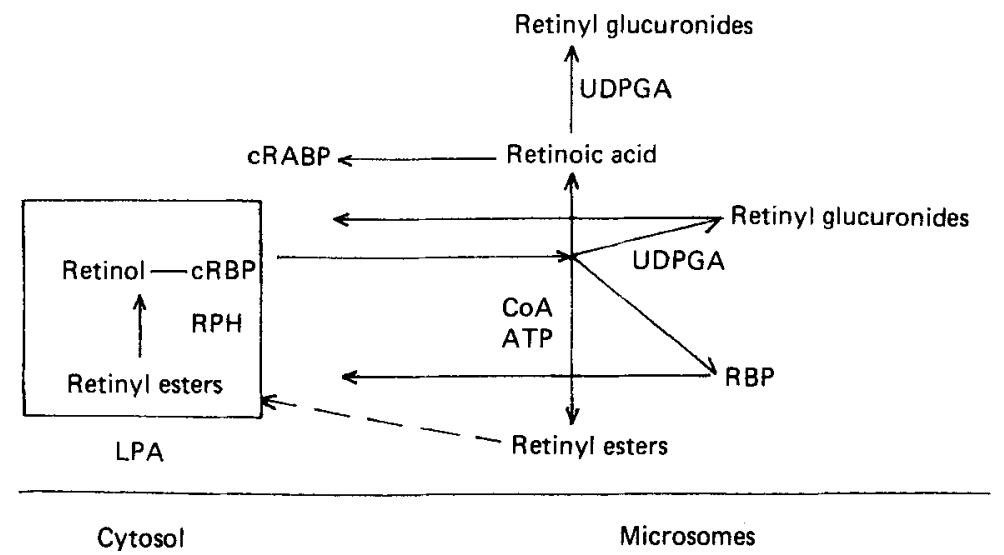

Fig. 5. A speculative scheme of some aspects of hepatic vitamin A metabolism. RPH, retinol palmitate palmitohydrolase (EC 3.1.1.21); cRBP, cellular retinol-binding protein; cRABP, cellular retinoicacid-binding protein; RBP, serum retinol-binding protein; LPA, lipid-protein aggregate; UDPGA, uridine diphosphate glucuronic acid.

The presence of carotene-cleavage activity has been recently reported in LPA in intestinal mucosa (Sklan, 1983), and a similar situation is shown here in the hepatic LPA. The mucosal carotene-cleavage activity appeared to be affected by a $\mathrm{Cu}-\mathrm{Zn}$-containing protein (Sklan, 1983). An additional involvement of $Z n$ in carotene cleavage is indicated by the demonstration in the LPA of alcohol dehydrogenase which is a $\mathrm{Zn}$-containing enzyme (Brand et al. 1975). It appears that alcohol dehydrogenase is associated with the LPA, but it is not clear if this and alcohol dehydrogenase (NAD $\left.(\mathrm{P})^{+}\right)$are two different enzymes.

Previously it has been suggested that the $\mathrm{Zn}$-containing enzymes, alcohol dehydrogenase and alcohol dehydrogenase (NAD(P) $)^{+}$, are identical in liver (Bliss, 1951), although this may not be the case in the eye (Lion et al. 1975; Bridges, 1977).

The present study has indicated several additional functions of vitamin A metabolism connected with the LPA; these include retinyl ester transport, holo-RBP release from the microsomes and carotene cleavage. However, following metabolism of retinol to retinoic acid in the microsomes, transport would seem to be independent of the LPA. Retinyl glucuronides are water soluble and are also apparently transported independently of the LPA. These observations together provide some support for the concept of a specific vitamin A metabolic function for the LPA.

\section{REFERENCES}

Adachi, N., Smith, J. E., Sklan, D. \& Goodman, D. S. (1982). Journal of Biological Chemistry 256, 9471-9475. Bliss, A. F. (1951). Archives of Biochemistry and Biophysics 31, 197-204.

Brand, L., Gohlke, J. R. \& Rao, D. S. (1975). Biochemistry 6, 3510-3512.

Bridges, C. D. B. (1977). Experimental Eye Research 24, 571-580.

Chen, C. C. \& Heller, J. (1979). Archives of Biochemistry and Biophysics 198, 572-579.

Chen, C. C., Heller, J., Ding, L. L. \& Horwitz, J. (1981). Archives of Biochemistry and Biophysics 207, $392-398$. Fidge, N. H., Smith, F. R. \& Goodman, D. S. (1969). Biochemical Journal 114, 689-694.

Goodman, D. S., Huang, H. S., Kanai, M. \& Shiratori, T. (1967). Journal of Biological Chemistry 242, 3543-3554. Heller, J. (1979). Archives of Biochemistry and Biophysics 198, 562-571.

Lion, F., Rotmans, J. P., Daemen, F. J. M. \& Bonting, S. L. (1975). Biochimica Biophysica Acta 384, $283-292$. Lippel, K. \& Olson, J. A. (1968). Journal of Lipid Research 9, 168-175.

Ong, D. E. \& Chytil, F. (1975). Journal of Biological Chemistry 250, 6113-6117.

Ong, D. E., Markert, C. \& Chui, J. (1978). Cancer Research 38, 4422-4426.

Ross, A. C. (1982). Journal of Biological Chemistry 257, 2453-2459. 
Sklan, D. (1983). British Journal of Nutrition 50, 417-425.

Sklan, D., Blaner, W., Adachi, N., Smith, J, E. \& Goodman, D. S. (1982). Archives of Biochemistry and Biophysics 214, 35-44.

Sklan, D. \& Donoghue, S. (1982a). British Journal of Nutrition 47, 273-280.

Sklan, D. \& Donoghue, S. (1982 b). Biochimica Biophysica Acta 711, 532-538.

Vallee, B. L. \& Hoch, F. L. (1955). Proceedings of the National Academy of Sciences, USA 41, 327-335. 